\title{
Quarter-symmetric metric connection on a Lorentzian $\alpha$-Sasakian manifold
}

\author{
Venkatesha and Divyashree $G$. \\ Department of Mathematics, Kuvempu University Jnanasahyadri Shankaraghatta-577451 Shivamogga Dist. Karnataka, India
}

Received: 19 October 2016, Accepted: 22 January 2017

Published online: 27 April 2017.

\begin{abstract}
In the present paper we study locally $\phi$-symmetric, locally projective $\phi$-symmetric, $\phi$-recurrent and $\phi$-projectively flat Lorentzian $\alpha$-Sasakian manifold with respect to quarter-symmetric metric connection. Further, the existence of a Lorentzian $\alpha$-Sasakian manifold admitting quarter-symmetric metric connection is shown by constructing an example.
\end{abstract}

Keywords: Lorentzian $\alpha$-Sasakian manifold, quarter-symmetric metric connection, locally $\phi$-symmetric, locally projective $\phi$-symmetric, $\phi$-recurrent, $\phi$-projectively flat.

\section{Introduction}

In 1924, the idea of semi-symmetric linear connection on a differentiable manifold was introduced by Friedmann and Schouten [5]. In 1930, Bartolotti [2] gave a geometrical meaning of such a connection. Further, the concept of metric connection with torsion on a Riemannian manifold was defined by Hayden [7]. Later Yano[18] studied some curvature and derivational conditions for semi-symmetric metric connection in Riemannian manifolds. As a generalization of semi-symmetric connection, Golab [6] introduced quarter symmetric connection on a differentiable manifold.

A linear connection $\tilde{\nabla}$ on an $n$-dimensional differentiable manifold is said to be a quarter-symmetric connection [6] if its torsion tensor $T$ is of the form

$$
T(X, Y)=\tilde{\nabla}_{X} Y-\tilde{\nabla}_{Y} X-[X, Y]=\eta(Y) \phi X-\eta(X) \phi Y
$$

where $\eta$ is a 1-form and $\phi$ is a tensor field of type (1). In particular, if we replace $\phi X$ by $X$ and $\phi Y$ by $Y$, then the quarter-symmetric connection reduces to a semisymmetric connection [5]. Further if quarter-symmetric linear connection $\tilde{\nabla}$ satisfies the condition

$$
\left(\tilde{\nabla}_{X} g\right)(Y, Z)=0
$$

for all $X, Y, Z \in \chi\left(M^{n}\right)$, where $\chi\left(M^{n}\right)$ is the Lie algebra of vector fields on a manifold $M$, then $\tilde{\nabla}$ is said to be a quarter-symmetric metric connection. Quarter-symmetric metric connection on different manifold have been studied by many authors like Rastogi [13,14], Mishra and Pandey [8], Yano and Imai [19], De et al. [3,9], Pradeep Kumar et al.[11, $12,16]$ and others.

In 2005, Yildiz and Murathan [21] introduced Lorentzian $\alpha$-Sasakian manifold. Later, many geometers have studied Lorentzian $\alpha$-Sasakian manifolds with different curvature restrictions in the papers [1,4,21,22] and others.

* Corresponding author e-mail: vensmath@gmail.com

(C) 2017 BISKA Bilisim Technology 
Motivated by the above studies, we have made an attempt to study quarter-symmetric metric connection on a Lorentzian $\alpha$-Sasakian manifold. The present paper is structured as follows: The section 2 is equipped with some preliminaries of Lorentzian $\alpha$-Sasakian manifold. Section 3 and 4 are devoted respectively to the study of locally $\phi$-symmetric and locally projective $\phi$-symmetric Lorentzian $\alpha$-Sasakian manifold admitting quarter-symmetric metric connection. And in sections 5 and 6, we have proved that $\phi$-recurrent and $\phi$-projectively flat Lorentzian $\alpha$-Sasakian manifolds with respect to quarter-symmetric metric connection is a generalized $\eta$-Einstein manifold. In the last section, we have constructed an example of a Lorentzian $\alpha$-Sasakian manifold with respect to quarter-symmetric metric connection.

\section{Preliminaries}

A n-dimensional differentiable manifold $\mathrm{M}$ is called a Lorentzian $\alpha$-Sasakian manifold [21], if it admits a (1) tensor field $\varphi$, a contravariant vector field $\xi$, a covariant vector field $\eta$ and a Lorentzian metric $g$ which satisfy,

$$
\begin{gathered}
\varphi^{2} X=X+\eta(X) \xi, g(X, \xi)=\eta(X), \eta(\xi)=-1 \\
g(\varphi X, \varphi Y)=g(X, Y)+\eta(X) \eta(Y) \\
\varphi \xi=0, \eta(\varphi X)=0
\end{gathered}
$$

Also a Lorentzian $\alpha$-Sasakian manifold $M$ satisfies [21,17],

$$
\begin{gathered}
\nabla_{X} \xi=\alpha \varphi X \\
\left(\nabla_{X} \eta\right)(Y)=\alpha g(\varphi X, Y), \\
\eta(R(X, Y) Z)=\alpha^{2}[g(Y, Z) \eta(X)-g(X, Z) \eta(Y)], \\
R(\xi, X) Y=\alpha^{2}[g(X, Y) \xi-\eta(Y) X], \\
R(\xi, X) \xi=\alpha^{2}[\eta(X) \xi+X], \\
R(X, Y) \xi=\alpha^{2}[\eta(Y) X-\eta(X) Y], \\
(X \varphi)(Y)=\alpha[g(X, Y) \xi+\eta(Y) X], \\
S(X, \xi)=(n-1) \alpha^{2} \eta(X), \\
\varphi Y)=S(X, Y)+(n-1) \alpha^{2} \eta(X) \eta(Y),
\end{gathered}
$$

for any vector fields $X, Y, Z \in M$ and where $\nabla$ denotes the operator of covariant differentiation with respect to Lorentzian metric $g$ and $\alpha \in R$. The relation between quarter-symmetric metric connection $\tilde{\nabla}$ and the Levi-Civita connection $\nabla$ on $M$ 
[10] is given by,

$$
\tilde{\nabla}_{X} Y=\nabla_{X} Y+\eta(Y) \phi X
$$

By virtue of (15), the relation between Riemannian curvature tensor $\tilde{R}$, Ricci tensor $\tilde{S}$ and scalar curvature $\tilde{r}$ with respect to quarter-symmetric metric connection and the Riemannian curvature tensor $R$, Ricci tensor $R$ and scalar curvature $r$ with respect to Levi-Civita connection in a Lorentzian $\alpha$-Sasakian manifold [10] are given as follows.

$$
\begin{gathered}
\tilde{R}(X, Y) Z=R(X, Y) Z+\alpha[g(\varphi X, Z) \varphi Y-g(\varphi Y, Z) \varphi X]+\alpha \eta(Z)[\eta(Y) X-\eta(X) Y] \\
\tilde{S}(Y, Z)=S(Y, Z)+\alpha[g(Y, Z)+n \eta(Y) \eta(Z)] \\
\tilde{r}=r .
\end{gathered}
$$

A Lorentzian $\alpha$-Sasakian manifold $M^{n}$ is said to be a generalized $\eta$-Einstein manifold [23] if the following condition

$$
S(X, Y)=\lambda g(X, Y)+\mu \eta(X) \eta(Y)+v \Omega(X, Y),
$$

holds on $\mathrm{M}$. Where $\lambda, \mu$ and $v$ are smooth functions and $\Omega(\mathrm{X}, \mathrm{Y})=\mathrm{g}(\varphi \mathrm{X}, \mathrm{Y})$. If $v=0$ then the manifold is an $\eta$ -Einstein manifold.

\section{Locally $\phi$-symmetric Lorentzian $\alpha$-Sasakian manifold admitting quarter-symmetric metric connection}

Definition 1. A Lorentzian $\alpha$-Sasakian manifold $M$ is said to be locally $\phi$-symmetric if

$$
\phi^{2}\left(\left(\nabla_{w} R\right)(X, Y) Z\right)=0
$$

for all vector fields $X, Y, Z$ and $W$ orthogonal to $\xi$ on $M$. This notion was introduced by Takahashi for Sasakian manifold

Definition 2. A Lorentzian $\alpha$-Sasakian manifold $M$ is said to be locally $\varphi$-symmetric with respect to quarter symmetric metric connection if

$$
\phi^{2}\left(\left(\tilde{\nabla}_{w} \tilde{R}\right)(X, Y) Z\right)=0
$$

for all vector fields $X, Y, Z$ and $W$ orthogonal to $\xi$ on $M$.

Theorem 1. A Lorentzian $\alpha$-Sasakian manifold is locally $\phi$-symmetric with respect to the quarter-symmetric metric connection $\tilde{\nabla}$ if and only if it is locally $\phi$-symmetric with respect to the Levi-Civita connection.

Proof.From equation (15) and (16), we have

$$
\left(\tilde{\nabla}_{w} \tilde{R}\right)(X, Y) Z=\left(\nabla_{w} \tilde{R}\right)(X, Y) Z+\eta(\tilde{R}(X, Y) Z) \phi W .
$$

Differentiating (16) covariantly with respect to $W$, we obtain

$$
\begin{aligned}
\left(\nabla_{w} \tilde{R}\right)(X, Y) Z & =\left(\nabla_{w} R\right)(X, Y) Z+\alpha\left\{g\left(\left(\nabla_{w} \phi\right)(X), Z\right) \phi Y+g(\phi X, Z)\left(\nabla_{w} \phi\right)(Y)\right\} \\
& \left.-\alpha\left\{g\left(\left(\nabla_{w} \phi\right)(Y), Z\right) \phi X\right\}+g(\phi Y, Z)\left(\nabla_{w} \phi\right)(X)\right\}+\alpha\left(\nabla_{w} \eta\right)(Z)[\eta(Y) X \\
& -\eta(X) Y]+\alpha\left\{\eta(Z)\left[\left(\nabla_{w} \eta\right)(Y) X-\left(\nabla_{w} \eta\right)(X) Y\right]\right\} .
\end{aligned}
$$


Using (7) and (12), (23) reduces to

$$
\begin{aligned}
\left(\nabla_{w} \tilde{R}\right)(X, Y) Z & =\left(\nabla_{w} R\right)(X, Y) Z-\alpha^{2}\{g(W, Y) \eta(Z)+g(W, Z) \eta(Y)\} \phi X \\
& +\alpha\{g(W, X) \eta(Z)+g(W, Z) \eta(X)\} \phi Y+\alpha^{2}\{g(\phi X, Z) g(W, Y) \xi+g(\phi X, Z) \eta(Y) W\} \\
& -\alpha^{2}\{g(\phi Y, Z) g(W, X) \xi+g(\phi Y, Z) \eta(X) W\}+\alpha^{2} g(\phi W, Z)[\eta(Y) X \\
& -\eta(X) Y]+\alpha^{2}[g(\phi W, Y) X-g(\phi W, X) Y] \eta(Z) .
\end{aligned}
$$

Taking inner product of (16) with $\mathrm{x}$ and then using (3), (5) and (8), we obtain

$$
\eta(\tilde{R}(X, Y) Z)=\alpha^{2}\{g(Y, Z) \eta(X)-g(X, Z) \eta(Y)\}
$$

Making use of equations (24) and (25), equation (22) becomes

$$
\begin{aligned}
\left(\tilde{\nabla}_{W} \tilde{R}\right)(X, Y) Z & =\left(\tilde{\nabla}_{W} R\right)(X, Y) Z-\alpha^{2}\{g(W, Y) \eta(Z)+g(W, Z) \eta(Y)\} \phi X \\
& +\alpha^{2}\{g(W, X) \eta(Z)+g(W, Z) \eta(X)\} \phi Y+\alpha^{2}\{g(\phi X, Z) g(W, Y) \xi+g(\phi X, Z) \eta(Y) W\} \\
& -\alpha^{2}\{g(\phi Y, Z) g(W, X) \xi+g(\phi Y, Z) \eta(X) W\}+\alpha^{2} g(\phi W, Z)[\eta(Y) X-\eta(X) Y]+\alpha^{2}[g(\phi W, Y) X \\
& -g(\phi W, X) Y] \eta(Z)+\alpha^{2}\{g(Y, Z) \eta(X)-g(X, Z) \eta(Y)\} \phi W .
\end{aligned}
$$

By applying $\phi^{2}$ on both sides of (26) and using (3), we obtain

$$
\begin{aligned}
\phi^{2}\left(\tilde{\nabla}_{W} \tilde{R}\right)(X, Y) Z & =\phi^{2}\left(\nabla_{W} R\right)(X, Y) Z-\alpha^{2}\{g(W, Y) \eta(Z)+g(W, Z) \eta(Y)\} \phi^{2}(\phi X)+\alpha^{2}\{g(W, X) \eta(Z) \\
& +g(W, Z) \eta(X)\} \phi^{2} \phi Y+\alpha^{2} g(\phi X, Z) \eta(Y) \phi^{2} W-\alpha^{2} g(\phi Y, Z) \eta(X) \phi^{2} W \\
& +\alpha^{2} g(\phi W, Z)[\eta(Y) X-\eta(X) Y]+\alpha^{2}[g(\phi W, Y) X+g(\phi W, Y) \eta(X) \xi-g(\phi W, X) Y \\
& -g(\phi W, X) \eta(Y) \xi] \eta(Z)+\alpha^{2}\{g(Y, Z) \eta(X)-g(X, Z) \eta(Y)\} \phi^{2} \phi W .
\end{aligned}
$$

Assuming $X, Y, Z$ and $W$ are orthogonal to $\xi,(27)$ reduces to,

$$
\phi^{2}\left(\tilde{\nabla}_{W} \tilde{R}\right)(X, Y) Z=\phi^{2}\left(\nabla_{W} R\right)(X, Y) Z
$$

This completes the proof of the theorem.

\section{Locally projective $\phi$-symmetric Lorentzian $\alpha$-Sasakian manifold admitting quarter-symmetric metric connection}

Definition 3. An n-dimensional Lorentzian $\alpha$-Sasakian manifold $M$ is said to be locally projective $\phi$-symmetric if

$$
\phi^{2}\left(\left(\tilde{\nabla}_{W} P\right)(X, Y) Z\right)=0
$$

for all vector fields $X, Y, Z, W$ orthogonal to $\xi$ and the projective curvature tensor $P$ [20] is given by

$$
P(X, Y) Z=R(X, Y) Z-\frac{1}{n-1}-[S(Y, Z) X-S(X, Z) Y]
$$

Definition 4. An n-dimensional Lorentzian $\alpha$-Sasakian manifold $M$ is said to be locally projective $\phi$-symmetric with respect to quarter-symmetric metric connection if

$$
\phi^{2}\left(\left(\tilde{\nabla}_{W} \tilde{P}\right)(X, Y) Z\right)=0
$$


for all vector fields $X, Y, Z$ and $W$ orthogonal to $\xi$. Here $\tilde{P}$ is the projective curvature tensor with respect to quartersymmetric metric connection given by

$$
\tilde{P}(X, Y) Z=\tilde{P}(X, Y) Z-\frac{1}{N-1}[\tilde{S}(Y, Z) X-\tilde{S}(X, Z) Y] .
$$

With the help of (16), we can write

$$
\left(\tilde{\nabla}_{W} \tilde{P}\right)(X, Y) Z=\left(\nabla_{W} \tilde{P}\right)(X, Y) Z+\eta(\tilde{P}(X, Y) Z) \phi W .
$$

Theorem 2. An n-dimensional Lorentzian $\alpha$-Sasakian manifold $M$ is locally projective $\phi$-symmetric with respect to the quarter symmetric metric connection if and only if $M$ is locally projective $\phi$-symmetric with respect to the Levi-Civita connection.

Proof. Differentiating (32) with respect to $W$, we get

$$
\left(\tilde{\nabla}_{W} \tilde{P}\right)(X, Y) Z=\left(\nabla_{W} \tilde{R}\right)(X, Y) Z-\frac{1}{n-1}\left[(\nabla W \tilde{S})(Y, Z) X-\left(\nabla_{W} \tilde{S}\right)(X, Z) Y\right] .
$$

In view of equations (17) and (24), (34) reduces to

$$
\begin{aligned}
\left(\nabla_{W} \tilde{P}\right)(X, Y) Z & =\left(\nabla_{W} R\right)(X, Y) Z-\alpha^{2}\{g(W, Y) \eta(Z)+g(W, Z) \eta(Y)\} \phi X \\
& +\alpha^{2}\{g(W, X) \eta(Z)+g(W, Z) \eta(X)\} \phi Y+\alpha^{2}\{g(\phi X, Z) g(W, Y) \xi \\
& +g(\phi X, Z) \eta(Y) W\}-\alpha^{2}\{g(\phi Y, Z) g(W, X) \xi+g(\phi Y, Z) \eta(X) W\} \\
& +\alpha^{2} g(\phi W, Z)[\eta(Y) X-\eta(X) Y]+\alpha^{2}[g(\phi W, Y) X-g(\phi W, X) Y] \eta(Z) \\
& \left.+\frac{\alpha^{2} n}{n-1}[g(\varphi W, Z) \eta(Y) X-\eta(X) Y\}+g(\phi W, Y) X g(\phi W, X) Y \eta(Z)\right] \\
& -\frac{1}{n-1}\left[\left(\nabla_{W} S\right)(Y, Z) X-\left(\nabla_{W} S\right)(X, Z) Y\right] .
\end{aligned}
$$

Substituting (32) in (35), we get

$$
\begin{aligned}
\left(\nabla_{W} \tilde{P}\right)(X, Y) Z & =\left(\nabla_{W} P\right)(X, Y) Z-\alpha^{2}\{g(W, Y) \eta(Z)+g(W, Z) \eta(Y)\} \varphi X \\
& +\alpha^{2}\{g(W, X) \eta(Z)+g(W, Z) \eta(X)\} \phi Y+\alpha^{2}\{g(\phi X, Z) g(W, Y) \xi \\
& +g(\phi X, Z) \eta(Y) W\}-\alpha^{2}\{g(\phi Y, Z) g(W, X) \xi+g(\phi Y, Z) \eta(X) W\} \\
& +\alpha^{2} g(\phi W, Z)[\eta(Y) X-\eta(X) Y]+\alpha^{2}[g(\varphi W, Y) X-g(\phi W, X) Y] \eta(Z) \\
& +\frac{\alpha^{2} n}{n-1}[g(\phi W, Z)\{\eta(Y) X-\eta(X) Y\}+\{g(\phi W, Y) X-g(\phi W, X) Y\} \eta(Z)] .
\end{aligned}
$$

By using the equations (16) and (17) in (32), we obtain

$$
\begin{aligned}
\tilde{P}(X, Y) Z & =R(X, Y) Z+\alpha[g(\phi X, Z) \phi Y-g(\phi Y, Z) \phi X]-\frac{1}{n-1}[S(Y, Z) X-(X, Z) Y] \\
& -\frac{\alpha}{n-1}[g(Y, Z) X-g(X, Z) Y]-\frac{\alpha}{n-1}[\eta(Y) X-\eta(X) Y] \eta(Z) .
\end{aligned}
$$

In virtue of (30), (37) becomes

$$
\begin{aligned}
\tilde{P}(X, Y) Z & =P(X, Y) Z+\alpha[g(\phi X, Z) \phi Y-g(\phi Y, Z) \phi X]-\frac{\alpha}{n-1}[g(Y, Z) X-g(X, Z) Y] \\
& -\frac{\alpha}{n-1}[\eta(Y) X-\eta(X) Y] \eta(Z) .
\end{aligned}
$$

Taking inner product of (37) with $\xi$ and then using (3),(5) and (8), we get

$$
\eta(\tilde{P}(X, Y) Z)=\left(\alpha^{2}-\frac{\alpha}{n-1}\right)[g(Y, Z) \eta(X)-g(X, Z) \eta(Y)]-\frac{1}{n-1}[S(Y, Z) \eta(X)-S(X, Z) \eta(Y)] .
$$


Now using equations (36) and (39), (33) reduces to

$$
\begin{aligned}
\left(\tilde{\nabla}_{W} \tilde{P}\right)(X, Y) Z & =\left(\nabla_{W} P\right)(X, Y) Z-\alpha^{2}\{g(W, Y) \eta(Z)+g(W, Z) \eta(Y)\} \varphi X \\
& +\alpha^{2}\{g(W, X) \eta(Z)+g(W, Z) \eta(X)\} \phi Y+\alpha^{2}\{g(\phi X, Z) g(W, Y) \xi \\
& +g(\phi X, Z) \eta(Y) W\}-\alpha^{2}\{g(\phi Y, Z) g(W, X) \xi+g(\phi Y, Z) \eta(X) W\} \\
& +\alpha^{2} g(\phi W, Z)[\eta(Y) X-\eta(X) Y]+\alpha^{2}[g(\phi W, Y) X-g(\phi W, X) Y] \eta(Z) \\
& +\frac{\alpha^{2} n}{n-1}[g(\phi W, Z)\{\eta(Y) X-\eta(X) Y\}+\{g(\phi W, Y) X-g(\phi W, X) Y\} \eta(Z)] \\
& +\left(\alpha^{2}-\frac{\alpha}{n-1}\right)[g(Y, Z) \eta(X)-g(X, Z) \eta(Y)] \phi W-\frac{1}{n-1}[S(Y, Z) \eta(X) \\
& -S(X, Z) \eta(Y)] \varphi W .
\end{aligned}
$$

By applying $\phi^{2}$ on both sides of (40) and assuming that $X, Y, Z$ and $W$ are orthogonal to $\xi$, we get

$$
\phi^{2}\left(\left(\tilde{\nabla}_{W} \tilde{P}\right)(X, Y) Z\right)=\phi^{2}\left(\left(\nabla_{W} P\right)(X, Y) Z\right)
$$

Hence the proof.

\section{$5 \phi$-Recurrent Lorentzian $\alpha$-Sasakian manifold admitting quarter-symmetric metric connection}

Definition 5. An n-dimensional Lorentzian $\alpha$-Sasakian manifold $M$ is said to be $\phi$-recurrent if there exists a non-zero 1-form A such that

$$
\phi^{2}\left(\left(\nabla_{W} P\right)(X, Y) Z\right)=A(W) R(X, Y) Z
$$

for arbitrary vector fields $X, Y, Z$ and $M$. If the 1-form A vanishes, then the manifold reduces to a $\phi$-symmetric manifold [15].

Definition 6. An n-dimensional Lorentzian $\alpha$-Sasakian manifold $M$ is said to be $\phi$-recurrent with respect to quartersymmetric metric connection if there exists a non-zero 1-form A such that

$$
\phi^{2}\left(\left(\tilde{\nabla}_{W} \tilde{R}\right)(X, Y) Z\right)=A(W) \tilde{R}(X, Y) Z
$$

for arbitrary vector fields $X, Y, Z$ and $W$.

Theorem 3. If a Lorentzian $\alpha$-Sasakian manifold is $\phi$-recurrent with respect to the quarter-symmetric metric connection then the manifold is a generalized $\eta$-Einstein manifold with respect to the Levi-Civita connection.

Proof. Suppose $M$ is $\phi$-recurrent with respect to quarter-symmetric metric connection. Then in view of (3), we can write (43) as

$$
\left(\tilde{\nabla}_{W} \tilde{R}\right)(X, Y) Z+\eta\left(\left(\tilde{\nabla}_{W} \tilde{R}\right)(X, Y) Z\right) \xi=A(W)(\tilde{R}(X, Y) Z)
$$

Taking inner product of (44) with $U$, we get

$$
g\left(\left(\tilde{\nabla}_{W} \tilde{R}\right)(X, Y) Z, U\right)+\eta\left(\left(\tilde{\nabla}_{W} \tilde{R}\right)(X, Y) Z\right) \eta(U)=A(W) g(\tilde{R}(X, Y) Z, U) .
$$

Using (22) in (45), we get

$$
g\left(\left(\tilde{\nabla}_{W} \tilde{R}\right)(X, Y) Z, U\right)+\eta\left(\left(\tilde{\nabla}_{W} \tilde{R}\right)(X, Y) Z\right) \eta(U)+\eta(\tilde{R}(X, Y) Z) g(\phi W, U)=A(W) g(\tilde{R}(X, Y) Z, U)
$$


Making use of (16), (24) and (25) in (46), we obtain

$$
\begin{aligned}
& g\left(\left(\nabla_{W} R\right)(X, Y) Z, U\right)-\alpha^{2}\{g(W, Y) \eta(Z)+g(W, Z) \eta(Y)\} g(\phi X, U)+\alpha^{2}\{g(W, X) \eta(Z)+g(W, Z) \eta(X)\} g(\phi Y, U) \\
& +\alpha^{2}\{g(\phi X, Z) g(W, Y) \eta(U)+g(\phi X, Z) \eta(Y) g(W, U)\}-\alpha^{2}\{g(\phi Y, Z) g(W, X) \eta(U)+g(\phi Y, Z) \eta(X) g(W, U)\} \\
& +\alpha^{2} g(\phi W, Z)[\eta(Y) g(X, U)-\eta(X) g(Y, U)]+\alpha^{2}[g(\phi W, Y) g(X, U)-g(\phi W, X) g(Y, U)] \eta(Z) \\
& +\eta\left(\left(\nabla_{W} R\right)(X, Y) Z\right) \eta(U)+\alpha^{2}\{g(\phi X, Z) \eta(Y) \eta(W)-g(\phi X, Z) g(W, Y)\} \eta(U)-\alpha^{2}\{g(\phi Y, Z) \eta(X) \eta(W) \\
& -g(\phi Y, Z) g(W, X)\} \eta(U)+\alpha^{2}\{g(\phi W, Y) \eta(Z) \eta(X)-g(\phi W, X) \eta(Y) \eta(Z)\} \eta(U)+\alpha^{2}\{g(Y, Z) \eta(X) \\
& -g(X, Z) \eta(Y)\} g(\phi W, U)=A(W) g(R(X, Y) Z, U)+\alpha A(W)\{g(\phi X, Z) g(\phi Y, U)-g(\phi Y, Z) g(\phi X, U) \\
& +g(X, U) \eta(Y) \eta(Z)-g(Y, U) \eta(X) \eta(Z)\}
\end{aligned}
$$

Plugging $Z=\xi$ in (47) and then using equations (3) and (5), we get

$$
\begin{aligned}
& g((\nabla W R)(X, Y) \xi, U)+\alpha^{2}\{g(W, Y)-\eta(W) \eta(Y)\} g(\phi X, U)-\alpha^{2}\{g(W, X) \\
& -\eta(W) \eta(X)\} g(\phi Y, U)-\alpha^{2}\{g(\phi W, Y) g(X, U)-g(\phi W, X) g(Y, U)\} \\
& +\eta((\nabla W R)(X, Y) \xi) \eta(U)+\alpha^{2}\{g(\phi W, X) \eta(Y)-g(\phi W, Y) \eta(X)\} \eta(U) \\
& =A(W) g(R(X, Y) \xi, U)-\alpha A(W)\{g(X, U) \eta(Y)-g(Y, U) \eta(X)\} .
\end{aligned}
$$

Taking $X=U=e_{i}$ in (48) and summing over $i, 1 \leq i \leq n$, we get

$$
\begin{aligned}
& \nabla_{W} S(Y, \xi)+\sum_{i=1}^{n-1} g\left(\left(\nabla_{W} R\right)\left(e_{i}, Y\right) \xi, \xi\right) g\left(e_{i}, \xi\right)+\alpha^{2} \operatorname{trace} \phi\{g(W, Y)-\eta(W) \eta(Y)\} \\
& -\alpha^{2}(n-1) g(\phi W, Y)=A(W) S(Y, \xi)-\alpha(n-1) A(W) \eta(Y)
\end{aligned}
$$

We denote the second term of the left hand side of the above equation by $G$. In this case $G$ vanishes. Therefore (49) reduces to

$$
\begin{aligned}
\nabla_{W} S(Y, \xi) & =-\alpha^{2} \operatorname{trace} \phi\{g(W, Y)-\eta(W) \eta(Y)\}+\alpha^{2}(n-1) g(\phi W, Y) \\
& +A(W) S(Y, \xi)-\alpha(n-1) A(W) \eta(Y)
\end{aligned}
$$

We have

$$
\left(\nabla_{W} S\right)(Y, \xi)=\nabla_{W} S(Y, \xi)-S\left(\nabla_{W} Y, \xi\right)-S\left(Y, \nabla_{W} \xi\right)
$$

Using (6), (7) and (13) in the above equation, we find

$$
\left(\nabla_{W} S\right)(Y, \xi)=(n-1) \alpha^{3} g(\phi W, Y)-\alpha S(\alpha W, Y)
$$

Equating (50) and (51), we obtain

$$
\begin{aligned}
(n-1) \alpha^{3} g(\phi W, Y)-\alpha S(\phi W, Y) & =-\alpha^{2} \operatorname{trace} \phi\{g(W, Y)-\eta(W) \eta(Y)\}+\alpha^{2}(n-1) g(\phi W, Y)+A(W) S(Y, \xi) \\
& -\alpha(n-1) A(W) \eta(Y) .
\end{aligned}
$$

Replacing $Y$ by $\phi Y$ in (52) and then using (4) and (14), we get

$$
S(Y, W)=\lambda g(Y, W)+\mu \eta(Y) \eta(W)+v g(W, \phi Y)
$$

where $\lambda=(n-1)(\alpha 2-\alpha), \mu=(1-n) \alpha$ and $v=\alpha$ trace $\phi$. Thus, the proof of the theorem completes. 


\section{$6 \phi$-projectively flat Lorentzian $\alpha$-Sasakian manifold admitting quarter-symmetric metric connection}

Definition 7. An n-dimensional Lorentzian $\alpha$-Sasakian manifold is said to be $\phi$-projectively flat with respect to quartersymmetric metric connection if it satisfies

$$
\phi^{2}(\tilde{P}(\phi X, \phi Y) \phi Z)=0
$$

Let $M$ be $\phi$-projectively flat with respect to quarter-symmetric metric connection. Then it is easy to see that $\phi^{2}(\tilde{P}(\phi X, \phi Y) \phi Z)=$ Oholds if and only if

$$
g(\tilde{P}(\phi X, \phi Y) \phi W)=0
$$

for every $X, Y, Z, W \in T M$.

Theorem 4. An n-dimensional $\phi$-projectively flat Lorentzian $\alpha$-Sasakian manifold admitting the quarter-symmetric metric connection is a generalized $\eta$-Einstein manifold.

Proof. Suppose $M$ is $\phi$-projectively flat with respect to quarter symmetric metric connection. Then we have from (32) that

$$
g(\tilde{R}(\phi X, \phi Y) \phi Z, \phi W)=\frac{1}{n-1}[\tilde{S}(\phi Y, \phi Z) g(\phi X, \phi W)-\tilde{S}(\phi X, \phi Z) g(\phi Y, \phi W)] .
$$

By using (3), (5), (16) and (17) in (56), we get

$$
\begin{aligned}
g(R(\phi X, \phi Y) \phi Z, \phi W) & +\alpha[g(X, \phi Z) g(Y, \phi W)-g(Y, \phi Z) g(X, \phi W)]=\frac{1}{n-1}[S(\phi Y, \phi Z) g(\phi X, \phi W) \\
& +\alpha g(\phi Y, \phi Z) g(\phi X, \phi W)-S(\phi X, \phi Z) g(\phi Y, \phi W)-\alpha g(\phi X, \phi Z) g(\phi Y, \phi W)]
\end{aligned}
$$

Let $\left\{e_{1}, e_{2}, \ldots, e_{n-1}, \xi\right\}$ be a local orthonormal basis of vector fields in $M$. Using the fact that $\left\{\phi e_{1}, \phi e_{2}, \ldots ., \phi e_{n-1}, \xi\right\}$ is also a local orthonormal basis, if we put $X=W=e_{i}$ in (57) and summing over $i$, we get

$$
\begin{aligned}
& \sum_{i=1}^{n-1} g\left(R\left(\phi e_{i}, \phi Y\right) \phi Z, \phi e_{i}\right)+\alpha\left[g\left(e_{i}, \phi Z\right) g\left(Y, \phi e_{i}\right)-g(Y, \phi Z) g\left(e_{i}, \phi e_{i}\right)\right]=\frac{1}{n-1} \sum_{i=1}^{n-1}\left[S(\phi Y, \phi Z) g\left(\phi e_{i}, \phi e_{i}\right)\right. \\
& \left.+\alpha g(\phi Y, \phi Z) g\left(\phi e_{i}, \phi e_{i}\right)-S\left(\phi e_{i}, \phi Z\right) g\left(\phi Y, \phi e_{i}\right)-\alpha g\left(\phi e_{i}, \phi Z\right) g\left(\phi Y, \phi e_{i}\right)\right]
\end{aligned}
$$

It is verified that[22]

$$
\begin{aligned}
& \sum_{i=1}^{n-1} g\left(R\left(\phi e_{i}, \phi Y\right) \phi Z, \phi e_{i}\right)=S(\phi Y, \phi Z)+g(\phi Y, \phi Z), \\
& \sum_{i=1}^{n-1} S\left(\phi e_{i}, \phi e_{i}\right)=r-(n-1) \alpha^{2}, \\
& \sum_{i=1}^{n-1} g\left(\phi e_{i}, \phi Z\right) S\left(\phi Y, \phi e_{i}\right)=S(\phi Y, \phi Z), \\
& \sum_{i=1}^{n-1} g\left(\phi e_{i}, \phi e_{i}\right)=n-1, \\
& \sum_{i=1}^{n-1} g\left(\phi e_{i}, \phi Z\right) g\left(\phi Y, \phi e_{i}\right)=g(\phi Y, \phi Z) .
\end{aligned}
$$


Making use of the equations (59)-(63), (58) becomes

$$
S(\phi Y, \phi Z)=\{-\alpha-n+1\} g(\phi Y, \phi Z)+\alpha(n-1) \operatorname{trace} \varphi g(Y, \phi Z) .
$$

Applying equations (4) and (14) in (64), we finally obtain

$$
S(Y, Z)=\lambda g(Y, Z)+\mu \eta(Y) \eta(Z)+v g(Y, \phi Z)
$$

where $\lambda=\{-\alpha-n+1\}, \mu=\{(-(n-1)(\alpha 2+1)-\alpha\}$ and $v=\alpha(n-1)$ trace $\varphi$. This completes the proof.

\section{Example of a Lorentzian $\alpha$-Sasakian manifold admitting quarter-symmetric metric connection}

Example 1. In this section, we consider a 3-dimensional manifold $M=\left\{(x, y, z) \in R^{3}\right\}$, where $(x, y, z)$ are the standard coordinates in $R^{3}$. We choose the vector fields

$$
e_{1}=e^{z} \frac{\partial}{\partial y}, e_{2}=e^{z}\left(\frac{\partial}{\partial x}+\frac{\partial}{\partial y}\right), e_{3}=\alpha \frac{\partial}{\partial z},
$$

which are linearly independent at each point of $M$ and $\alpha$ is constant. Let $g$ be a Lorentzian metric defined by

$$
\begin{aligned}
& g\left(e_{1}, e_{3}\right)=g\left(e_{2}, e_{3}\right)=g\left(e_{1}, e_{2}\right)=0, \\
& g\left(e_{1}, e_{1}\right)=g\left(e_{2}, e_{2}\right)=1, g\left(e_{3}, e_{3}\right)=-1
\end{aligned}
$$

Let $\eta$ be a 1-form defined by

$$
\eta(Z)=g\left(Z, e_{3}\right)
$$

for any $Z \in \chi(M)$. Let $\phi$ be the $(1,1)$ tensor field defined by

$$
\phi e_{1}=-e_{1}, \phi e_{2}=-e_{2}, \phi e_{3}=0
$$

Using the linearity of $\phi$ and $g$, we have

$$
\eta\left(e_{3}\right)=-1, \phi^{2} Z=Z+\eta(Z) e_{3}, g(\phi Z, \phi W)=g(Z, W)+\eta(Z) \eta(W),
$$

For any $Z, W \in \chi(M)$. Then we have

$$
\left[e_{1}, e_{2}\right]=0,\left[e_{1}, e_{3}\right]=-\alpha e_{1},\left[e_{2}, e_{3}\right]=-\alpha e_{2}
$$

Using the Koszul's formula, we have

$$
\begin{array}{r}
\nabla_{e_{1}} e_{1}=-\alpha e_{1}, \nabla_{e_{1}} e_{2}=0, \nabla_{e_{1}} e_{3}=-\alpha e_{1}, \\
\nabla_{e_{2}} e_{1}=0, \nabla_{e_{2}} e_{2}=-\alpha e_{3}, \nabla_{e_{2}} e_{3}=-\alpha e_{2}, \\
\nabla_{e_{3}} e_{1}=0, \nabla_{e_{3}} e_{2}=0, \nabla_{e_{3}} e_{3}=0 .
\end{array}
$$


By using (15) in the above equation, we obtain

$$
\begin{aligned}
& \tilde{\nabla}_{e_{1} e_{1}}=-\alpha e_{3}, \tilde{\nabla}_{e_{1} e_{2}=0, \tilde{\nabla}_{e_{1}} e_{3}=(\alpha-1) e_{1},}, \\
& \tilde{\nabla}_{e_{2}} e_{1}=0, \tilde{\nabla}_{e_{2}} e_{2}=-\alpha e_{3}, \tilde{\nabla}_{e_{2}} e_{3}=(\alpha-1) e_{2}, \\
& \tilde{\nabla}_{e_{3}} e_{1}=0, \tilde{\nabla}_{e_{3}} e_{2}=0, \tilde{\nabla}_{e_{3}} e_{3}=0 .
\end{aligned}
$$

Using (1), the torsion tensor $\tilde{T}$ of the quarter symmetric metric connection $\tilde{\nabla}$ may be expressed as follows.

$$
\tilde{T}\left(e_{i}, e_{i}\right)=0, \text { for } i=1,2,3, \quad \tilde{T}\left(e_{1}, e_{2}\right)=0, \tilde{T}\left(e_{1}, e_{3}\right)=e_{1}, \quad \tilde{T}\left(e_{1}, e_{3}\right)=e_{2}
$$

Also,

$$
\left(\tilde{\nabla} e_{1} g\right)\left(e_{2}, e_{3}\right)=\left(\tilde{\nabla} e_{2} g\right)\left(e_{3}, e_{1}\right)=\left(\tilde{\nabla} e_{3} g\right)\left(e_{2}, e_{1}\right)=0 .
$$

Hence, we proved the existence of a Lorentzian $\alpha$-Sasakian manifold admitting quarter symmetric metric connection through an example.

\section{Acknowledgement}

The second author is thankful to UGC for financial support in the form of Rajiv Gandhi National Fellowship (F1-17.1/2015-16/RGNF-2015-17-SC-KAR-26367). The authors are thankful to the referees for their valuable suggestions and remarks in the improvement of the paper.

\section{Competing interests}

The authors declare that they have no competing interests.

\section{Authors' contributions}

All authors have contributed to all parts of the article. All authors read and approved the final manuscript.

\section{References}

[1] Ajit Barman, On Lorentzian $\alpha$-Sasakian manifolds admitting a type of semi-symmetric metric connection, Novi Sad J. Math., 44(2), 77-88, (2014).

[2] E. Bartolotti,Sulla geometria della variata a connection affine,Ann. di Mat. 4(8), 53-101, (1930).

[3] U. C. De and J. Sengupta, Quarter-symmetric metric connection on a Sasakian manifold,Communications de la Faculte des Sciences de l'Universite dAnkara., 49(1-2), 7-13, (2000).

[4] S. Dey and A. Bhattacharyya, Some properties of Lorentzian al pha-Sasakian manifolds with respect to quarter-symmetric metric connection,Acta Univ. Palacki. Olomuc., Fac. rer. nat., Mathematica 54, 2, 21-40, (2015).

[5] A. Friedmann and J. A. Schouten, Uber die Geometrie der halbsymmetrischen Ubertragungen,Mathematische Zeitschrift., 21(1) 211223, (1924).

[6] S. Golab,On semi-symmetric and quarter-symmetric linear connections, The Tensor Society., 22(3), 293-301,(1975).

[7] H. A. Hayden,Subspaces of a space with torsion,Proceedings London Mathematical Society., 34, 27-50, (1932).

[8] R. S. Mishra and S. N. Pandey, On quarter-connections metric F-connections, The Tensor Society., 34(1), 1-7, (1980).

[9] A. K. Mondal and U. C. De, Some properties of a quarter-symmetric metric connection on a Sasakian manifold, Bulletin of Mathematical Analysis and Applications., 1(3), 99-108, (2009). 
[10] C. Patra and A. Bhattacharyya,Quarter-symmetric metric connection on pseudosymmetric Lorentzian $\alpha$-Sasakian manifolds, International J.Math. Combin. 1, 46-59, (2013).

[11] K. T. Pradeep Kumar, C. S. Bagewadi, and Venkatesha, On projective ö-symmetric K-contact manifold admitting quartersymmetric metric connection, Differential Geometry Dynamical Systems., 13, 128-137, (2011).

[12] K. T. Pradeep Kumar, Venkatesha, and C.S.Bagewadi,On $\varphi$-Recurrent Para-Sasakian Manifold Admitting Quarter-Symmetric, Metric Connection,ISRN Geometry., Article ID 317253, 10 pages doi:10.5402, (2012).

[13] S. C. Rastogi, On quarter-symmetric metric connection,Comptes Rendus de l'Academie Bulgare des Sciences., 31(7), 811-814, (1978).

[14] S. C. Rastogi, On quarter-symmetric metric connections, The Tensor Society, vol. 44, no. 2, pp. 133 141, (1987).

[15] T. Takahashi, Sasakian $\varphi$-symmetric spaces,Tohoku math J., 29, 91-113, (1977).

[16] Venkatesha, K.T.P. Kumar and C.S. Bagewadi, On Quarter-Symmetric Metric Connection in a Lorentzian Para-Sasakian, Manifold, Azerbaijan Journal of Mathematics., 5(1), 3-12, (2015).

[17] S. Yadav and D. L. Suthar, Certain derivation on Lorentzian $\alpha$-Sasakian manifolds,Mathematics and Decision Science 12 (2012).

[18] K. Yano, On semi-symmetric metric connection, Revue Roumaine de Mathematiques Pures et Appliqu'ees., 15, 1579-1586, (1970).

[19] K. Yano and T. Imai, Quarter-symmetric metric connections and their curvature tensors, The Tensor Society., 38, 13-18, (1982).

[20] K. Yano and M. Kon, Structures on manifolds, volume 3 of Series in Pure Mathematics, World Scientific Publishing Co.,Singapore, (1984).

[21] Yildiz A, Murathan C, On Lorentzian $\alpha$-Sasakian manifolds, Kyungpook Math. J. 45, 95-103, (2005).

[22] A. Yildiz and M. Turan,A Class of Lorentzian $\alpha$-Sasakian Manifolds,Kyungpook Math. J. 49, 789-799, (2009).

[23] A. Yildiz, U. C. De and Erhan Ata,On a type of Lorentzian $\alpha$-Sasakian manifolds,MATH. REPORTS, 16(66), 1, 61-67, (2014). 